\title{
CONDUCTAS SEXUALES ONLINE Y SUS MOTIVACIONES: UNA APROXIMACIÓN DESDE LA DIVERSIDAD SEXUAL
}

\author{
Jesús Castro-Calvo \\ Dpto. Personalidad, Evaluación y Tratamientos Psicológicos \\ Universitat de València \\ jesus.castro@uv.es \\ Rafael Ballester-Arnal \\ Dpto. Psicología Básica, Clínica y Psicobiología \\ Universitat Jaume I, Castellón \\ María Dolores Gil-Llario \\ Dpto. Psicología Evolutiva y de la Educación \\ Universitat de València, Estudi General \\ Marta García-Barba \\ Dpto. Psicología Básica, Clínica y Psicobiología \\ Universitat Jaume I, Castellón \\ Verónica Cervigón Carrasco \\ Dpto. Psicología Básica, Clínica y Psicobiología \\ Universitat Jaume I, Castellón
}

Recepción Artículo: 10 febrero 2020

Admisión Evaluación: 4 marzo 2020

Informe Evaluador 1: 13 marzo

Informe Evaluador 2: 17 marzo 2020

Aprobación Publicación: 20 abril 2020

Este trabajo se ha realizado gracias a tres proyectos de investigación financiados por la Universitat Jaume I de Castellón (P1.1B2012-49, P1.1B2015-82 y UJI-B2018-42).

\section{RESUMEN}

El uso de Internet con objetivos sexuales (cibersexo) es una actividad cada vez más generalizada en todos los sectores de la población. Sin embargo, el modo en el que se realiza (frecuencia, tipos de actividad sexual online-ASO-, motivaciones, etc.) puede cambiar en función de características como la orientación sexual. Cuando el hecho de sentir atracción sexual hacia personas del mismo sexo suponía mayor rechazo y estigmatización que en la actualidad, el cibersexo constituía un espacio seguro donde experimentar con la sexualidad; así, era fácil comprender que su consumo entre personas homosexuales y bisexuales fuera superior. Actualmente, su empleo como espacio seguro parece quedar en segundo plano, ganando protagonismo su uso como una forma plausible de conectar romántica y sexualmente con otras personas. El objetivo de este trabajo es analizar si ciertas dimensiones del consumo de cibersexo (su frecuencia, los tipos de ASO y los motivos para el consumo) varían en función de la orientación sexual. A tal fin, 553 personas entre los 18-40 años (54.2\% hombres, 45.8\% mujeres; $36.2 \%$ heterosexuales, $36.2 \%$ bisexuales, $27.7 \%$ homosexuales) completaron una encuesta online sobre consu- 
mo de cibersexo. En hombres, la orientación sexual (ser homosexual o bisexual) mostró gran influencia, principalmente en ASO que requieren contacto con otros usuarios (p.e., buscar pareja [ $p<.001]$ o usar la webcam con fines sexuales [ $p<.01]$ ) y las motivaciones sociales para hacerlo (p.e., usarlo para establecer relaciones [ $p=.001]$ ). En mujeres, la orientación sexual influyó de forma más limitada, y sobre algunas ASO concretas (insinuarse a otros usuarios [ $p<.01$ ] 0 usar la webcam con objetivo sexual [ $p<.001]$ ) 0 algunas motivaciones (como usarlo para relajarse del estrés [ $p=.01]$ ); en este caso, las mujeres bisexuales contestaban afirmativamente con mayor frecuencia. Estas diferencias se discuten en el contexto de la construcción actual de la diversidad sexual.

Palabras clave: cibersexo; actividad sexual online; motivaciones; orientación sexual

\section{ABSTRACT}

Online sexual activities and motives: an approach from the sexual diversity perspective. The use of the Internet for sexual purposes (AKA cybersex) is an increasingly widespread activity in all the population. However, the way in which it is conducted (frequency, types of online sexual activity -ASO-, motivations) may change depending on characteristics such as sexual orientation. When feeling sexual attraction towards people of the same sex supposed rejection and stigmatization, cybersex was a safe space where to experiment with sexuality; thus, it was easy to understand that their consumption among homosexual and bisexual people was higher. Currently, its use as a safe space seems to be in a second place, gaining prominence its use as a plausible way to connect romantically and sexually with other people. The objective of this work was to analyze if certain aspects of cybersex consumption (frequency, types of ASO and reasons for consumption) vary depending on sexual orientation. With this aim, 553 people between $18-40$ years old (54.2\% men, $45.8 \%$ women; $36.2 \%$ heterosexual, $36.2 \%$ bisexual, $27.7 \%$ homosexual) completed an online survey on cybersex consumption. In men, sexual orientation (being homosexual or bisexual) showed great influence, mainly in ASOs that require contact with other users (looking for a partner [ $p<.001]$ or using the webcam for sexual purposes [p<.01]) and the social motives for doing so (e.g., using it to establish relationships [ $p=.001])$. In women, influence of sexual orientation was limited to some specific ASO (insinuate other users [ $p<.01]$ or use the webcam for sexual purposes [ $p<.001]$ ) and some motives (such as using it to relax from stress [ $p=.01]$ ); In this case, bisexual women answered affirmatively more frequently. These differences are discussed in the context of the current construction of sexual diversity.

Keywords: online sexual activities; motives; sexual orientation

\section{INTRODUCCIÓN}

El cibersexo, definido como «el uso de Internet con objetivos de gratificación sexual» (Cooper \& GriffinShelley, 2002), comprende actividades que se pueden realizar o bien en solitario o bien mediante la interacción con otros usuarios online (Shaughnessy et al., 2011). Entre las Actividades Sexuales Online (ASO) que no requieren contacto con otros usuarios, destacarían la descarga y el visionado de pornografía (presumiblemente por la facilidad para acceder anónima y gratuitamente a este tipo de contenidos). La realización de esta ASO se acompaña habitualmente de la masturbación, de modo que la persona que la realiza suele obtener gratificación sexual inmediata asociada a este comportamiento. Entre las que sí implican la interacción con otros usuarios, deberíamos distinguir entre aquellas que también en ocasiones se acompañan de masturbación concurrente (como mantener conversaciones sexuales con otros usuarios -mediante chat o webcam- 0 intercambiar imágenes sexuales a través del móvil -Sexting-) y las que no. Entre estas últimas, destaca el empleo de aplicaciones móviles basadas en geolocalización (p.e., Tinder o Grindr) para conocer personas con las que iniciar una relación romántica o sexual. En este último caso, la gratificación sexual no se produciría durante la realización la ASO, sino a posteriori (cuando el usuario contacta offline con la persona que ha conocido).

Una de las principales motivaciones tras estos comportamientos es disponer de contenido sexual con el que masturbarse y alcanzar el placer sexual (Castro-Calvo et al., 2018). En este sentido, un estudio en consumidores de cibersexo hallaba que la mayoría de usuarios ( $80 \%$ en chicos, $73 \%$ en chicas) consideraba que su ASO satisfacía entre moderada y completamente su deseo sexual (Daneback et al., 2013). Sin embargo, esta no es la única 
motivación, y al hecho de alcanzar el placer sexual se le suman otros aspectos que explican la popularidad del cibersexo y las preferencias por una u otra ASO. De acuerdo con una reciente revisión de Castro et al. (2018), existen siete motivos -además del hedónico- por los que la gente consume cibersexo. El primero resultaría muy prosaico: la gente lo consume porque es gratis, accesible y anónimo. Otro motivo (sobre todo entre los más jóvenes) es por curiosidad: algunas personas buscan en la ASO un medio de satisfacer su curiosidad sexual, sobre todo para explorar preferencias o contenidos sexuales que se alejan de lo que acostumbran a hacer en sus relaciones offline (por ejemplo, esto motivaría el consumo de un adolescente con dudas sobre su orientación sexual que decide buscar pornografía homosexual para "autoanalizar" su reacción). Otro grupo de motivaciones tiene que ver con aspectos sociales, como el hecho de realizar una ASO por presión de una tercera persona (una motivación obviamente disfuncional y que puede tener un impacto muy negativo) 0, al contrario, para conocer a una persona con la que mantener relaciones románticas/sexuales online u offline. Esta última motivación es la que explica la popularidad del uso de los chats o las webcams para reproducir fantasías sexuales, o el empleo de apps para ligar. La última motivación tendría que ver con el uso de la ASO como una forma de regulación emocional: algunas personas encuentran en el cibersexo un modo de manejar sus emociones -sobre todo, el estrés o los estados de ánimo disfóricos-. Cuando sucede esporádicamente, no suele entrañar mayores consecuencias; sin embargo, el hecho de usar el cibersexo como la única -0 la principal- estrategia de afrontamiento aumentaría exponencialmente la probabilidad de desarrollar un consumo problemático de cibersexo (Lew-Starowicz et al., 2019).

Por otro lado, existen toda una serie de características individuales que modulan el comportamiento sexual online, entre las que destaca la orientación sexual. Un hallazgo sistemático de la mayoría de los estudios es que las personas homosexuales y las bisexuales consumirían más cibersexo, y además lo harían de forma más problemática. Este aumento en el consumo de cibersexo se aprecia en todas las ASO, pero en especial en aquellas que implican el contacto sexual con otros usuarios: así, las personas con una orientación alternativa a la heterosexual utilizarían en mucha mayor medida Internet para conocer y mantener relaciones sexuales con personas que comparten sus preferencias sexuales (Seal et al., 2015). Para explicar su popularidad, algunos recurren a la metáfora de Internet como «oasis erótico» (Ross et al., 2000, p. 750): en contextos en los que no ser heterosexual ha sido -y continua siendo - objeto de sanción social, Internet ha permitido que las personas homosexuales y bisexuales puedan acceder a contenidos y actividades sexuales ajustados a su preferencia. Estas personas encontrarían en Internet -y en el anonimato que garantiza- el entorno ideal donde comenzar a explorar su sexualidad sin temor al estigma que supondría hacerlo de forma pública, sobre todo en contextos con valores más tradicionales (p.e., en el ámbito rural) 0 donde pertenecer al colectivo LGTBI supondría discriminación, violencia verbal y/o física e incluso sanción legal. El acceso a información sexual relevante en este colectivo o a pornografía ajustada a sus preferencias constituirían hitos importantes, pero más destacado aún ha sido el papel que habría desempeñado Internet a la hora de facilitar encontrar pareja (romántica y/o sexual). Como dato ilustrativo, Rosser et al. (2009) afirmaban que el 99\% de su muestra de 1,024 Hombres que tenían Sexo con Hombres (HSH) utilizaba Internet para encontrar parejas. Entre los motivos que justifican esta popularidad, destacan: Io sencillo que resulta encontrar una pareja que comparta sus preferencias sexuales (evitando intentar ligar con alguien heterosexual), encontrar parejas que sólo quieran mantener relaciones sexuales (sin vinculación sentimental), la seguridad personal de que el contacto se realiza en un entorno privado y el control que te da el poder decidir si, tras un contacto online, se desea ver offline a la persona (Grov et al., 2008).

Estos estudios sugerirían que la orientación sexual condiciona la preferencia por distinto tipo de ASO, y que influye sobre las motivaciones que explican su comportamiento sexual online. Sin embargo, apenas disponemos de estudios que analicen en detalle esta cuestión. Otro problema es que la mayoría de estudios se han realizado en otros contextos culturales (principalmente, EEUU), lo que podría menoscabar su generalizabilidad. España es uno de los países con una actitud más positiva hacia la diversidad sexual (sólo por detrás de países como Suecia o Noruega) (Petrou \& Lemke, 2018); esto podría influir en la frecuencia y motivaciones por las que las personas 
con una orientación sexual alternativa a la heterosexual consumen cibersexo. Así, el objetivo con el que se planteó este estudio fue analizar si varios aspectos del consumo de cibersexo (concretamente, su frecuencia, las preferencias por distinta ASO y los motivos para el consumo) varían en función de la orientación sexual.

\section{MÉTODO}

\section{Participantes}

Este trabajo forma parte de un proyecto más amplio, el proyecto ADISEX, realizado por el grupo Salusex de la Universidad Jaume I de Castellón y la Universidad de Valencia. Para este artículo se han utilizado datos de un total de 553 personas de entre 18-40 años ( $M=24.73$; $D T=5.39$ ). El 54.2\% de los participantes eran hombres, y el $45.8 \%$ restante mujeres. En cuanto a la orientación sexual, el 36.2\% se definían como heterosexuales, el $36.2 \%$ como bisexuales y el $27.7 \%$ como homosexuales. El objetivo de esta investigación era comparar el comportamiento sexual online en función del sexo y la orientación sexual de la muestra, de modo que se clasificó a Ios participantes en 6 grupos de acuerdo a estas dos variables: hombres heterosexuales ( $n=100)$, bisexuales $(n=100)$ y homosexuales $(n=100)$ y mujeres heterosexuales $(n=100)$, bisexuales $(n=100)$ y homosexuales $(n=53)$. En la siguiente tabla, se detallan algunas de sus características sociodemográficas (Tabla 1):

Tabla 1. Características sociodemográficas en función del sexo y la orientación sexual

\begin{tabular}{|c|c|c|c|c|c|c|}
\hline & \multicolumn{3}{|c|}{ Hombres $(n=300)$} & \multicolumn{3}{|c|}{ Mujeres $(n=253)$} \\
\hline & $\begin{array}{c}\text { Heterosexua } \\
\text { les }\end{array}$ & $\begin{array}{l}\text { Bisexual } \\
\text { es }\end{array}$ & $\begin{array}{l}\text { Homosexu } \\
\text { ales }\end{array}$ & $\begin{array}{c}\text { Heterosexua } \\
\text { les }\end{array}$ & $\begin{array}{l}\text { Bisexual } \\
\text { es }\end{array}$ & $\begin{array}{l}\text { Homosexu } \\
\text { ales }\end{array}$ \\
\hline Edad media $(D T)$ & $27.2(6.0)$ & $\begin{array}{l}25.4 \\
(5.8)\end{array}$ & $25.8(5.6)$ & $23.1(4.1)$ & $\begin{array}{l}22.4 \\
(4.1)\end{array}$ & $23.7(4.1)$ \\
\hline Tipo de relación & & & & & & \\
\hline Sin pareja & $28 \%$ & $44 \%$ & $49 \%$ & $28 \%$ & $24 \%$ & $37.7 \%$ \\
\hline Pareja estable & $60 \%$ & $40 \%$ & $37 \%$ & $55 \%$ & $54 \%$ & $54.7 \%$ \\
\hline Parejas esporádicas & $12 \%$ & $16 \%$ & $14 \%$ & $17 \%$ & $22 \%$ & $7.5 \%$ \\
\hline Ordenador propio & $97 \%$ & $97 \%$ & $100 \%$ & $98 \%$ & $97 \%$ & $100 \%$ \\
\hline $\begin{array}{l}\text { Tiempo semanal } \\
\text { online en horas }(D T)\end{array}$ & $31.1(26.8)$ & $\begin{array}{c}26.1 \\
(26.6)\end{array}$ & $28.3(22.7)$ & $27.3(21.6)$ & $\begin{array}{c}35.7 \\
(27.2)\end{array}$ & $20.8(15.8)$ \\
\hline
\end{tabular}

\section{Instrumentos}

En esta investigación, se aplicó una batería de instrumentos de evaluación que permitían explorar múltiples aspectos del comportamiento sexual online y offline. El tiempo medio para completar todos los cuestionarios fue de 28,6 minutos (DT=42,6). En este trabajo, se analizaron únicamente las siguientes variables:

-Variables sociodemográficas: Ios participantes completaron un cuestionario Ad-Hoc en el que se les preguntaba su sexo, edad, relación de pareja, orientación sexual, si disponían de ordenador con acceso a Internet y el tiempo semanal online (en general).

-Comportamiento sexual online: empleamos el Cuestionario de Prácticas Sexuales Online (CPSO, SalusexUnisexsida, 2016) para explorar los siguientes aspectos: a) si alguna vez habían utilizado Internet con objetivo sexual (Sí/No); b) el tiempo dedicado actualmente a la ASO ("¿Cuántas horas semanales pasas conectado a páginas sexuales?'); c) el tipo de ASO que los participantes habían realizado (12 ítems dicotómicos) y los motivos detrás del consumo de cibersexo (8 ítems dicotómicos). Las ASO y motivos concretos evaluados se detallan en la tablas 2 y 3 . Todos ellos se extrajeron de estudios previos que exploraban estas dimensiones (Castro-Calvo et al., 2018; Wéry \& Billieux, 2017). La fiabilidad de la escala de ASO y de motivaciones fue de $\alpha=.76$ y $\alpha=63$ respectivamente. 


\section{Procedimiento}

Los participantes en esta investigación fueron reclutados y evaluados a través de ADISEX (http://adiccionalsexo.uji.es/), una plataforma que permite una evaluación completa del control de los impulsos sexuales. La página era accesible a través de cualquier motor de búsqueda mediante términos de búsqueda como: "Adicción al cibersexo", "Adicciones sexuales", "Evaluación de la adicción al cibersexo" o "Tratamiento de la adicción al cibersexo". A fin de enriquecer el perfil e incrementar el número de usuarios que accedían a la evaluación, se lanzó una campaña de difusión en la que se solicitaba colaboración en un estudio a través del sistema de publicidad de Facebook (publicaciones sugeridas). Este estudio ha sido aprobado por el comité de ética de la Universitat Jaume I de Castellón.

\section{Análisis de los datos}

Los datos se analizaron mediante el paquete estadístico SPSS Versión 25.0. Se realizaron análisis descriptivos para caracterizar a los participantes en términos sociodemográficos y de comportamiento sexual online, analizando posteriormente el papel modulador de la orientación sexual. Las diferencias en conducta sexual online en función de esta variable se evaluaron mediante la prueba ANOVA de un factor (variables continuas) y el contraste Chi Cuadrado (variables categóricas).

A fin de evitar que las diferencias entre el consumo de cibersexo de hombres y mujeres dificultaran el análisis de las diferencias en función de la orientación sexual, los análisis se realizaron para cada sexo por separado.

\section{RESULTADOS}

\section{Actividad sexual online en función de la orientación sexual (hombres)}

Como se aprecia en la tabla 2, la orientación sexual de los hombres no influyó sobre el hecho de haber usado alguna vez Internet con fines sexuales ni sobre el tiempo online dedicado a la actividad sexual. En cuanto a la prevalencia de distintas ASO, sí se aprecian diferencias significativas en nueve de ellas. Salvo alguna excepción (p.e., la lectura de textos eróticos), la prevalencia es mayor entre homosexuales, seguido de cerca por bisexuales y finalmente heterosexuales. Sería el caso del uso de Internet para buscar pareja sexual (realizado por el $78 \%$ de Ios homosexuales frente al $58 \%$ de bisexuales y el $40 \%$ de heterosexuales; $p<.001$ ) 0 del uso de las webcams para mantener relaciones con otros usuarios ( $69 \%, 55 \%$ y $44 \%$ respectivamente; $p<.001)$. En cuanto a las motivaciones para practicar cibersexo, la orientación influyó sólo en dos de ellas (en ambos casos, motivaciones "sociales"): su uso para conocer a otras personas ( $p<.001)$ y para intentar establecer relaciones ( $p=.001)$. En ambos casos, la prevalencia era mayor en hombres homosexuales, seguido de bisexuales y heterosexuales. 
Tabla 2. Comportamiento sexual online en función de la orientación sexual (hombres)

\begin{tabular}{|c|c|c|c|c|}
\hline & $\begin{array}{l}\text { Heterosexual } \\
\text { es }\end{array}$ & Bisexuales & $\begin{array}{c}\text { Homosexua } \\
\text { les }\end{array}$ & $\begin{array}{l}\text { Estadístico } \\
\text { diferencial }\end{array}$ \\
\hline $\begin{array}{l}\text { ¿Alguna vez has utilizado Internet con fines } \\
\text { sexuales? }\end{array}$ & $97.0 \%$ & $100 \%$ & $100 \%$ & $\chi^{2}=6.06$ \\
\hline $\begin{array}{l}\text { Tiempo medio dedicado a la ASO en } \\
\text { minutos }(D T)\end{array}$ & $\begin{array}{l}259.1 \\
(255.5)\end{array}$ & $\begin{array}{l}216.53 \\
(207.1)\end{array}$ & $\begin{array}{l}241.3 \\
(227.7)\end{array}$ & $f=0.821$ \\
\hline \multicolumn{5}{|l|}{ Tipos de actividad sexual online } \\
\hline $\begin{array}{l}\text { Visionado de imágenes o películas } \\
\text { pornográficas }\end{array}$ & $98.0 \%$ & $97.0 \%$ & $99.0 \%$ & $\chi^{2}=1.02$ \\
\hline $\begin{array}{l}\text { Búsqueda de información sobre } \\
\text { educación sexual }\end{array}$ & $75.0 \%$ & $88.0 \%$ & $87.0 \%$ & $\chi^{2}=7.53 *$ \\
\hline Lectura de textos eróticos & $55.0 \%$ & $75.0 \%$ & $72.0 \%$ & $\chi^{2}=10.57 * *$ \\
\hline Búsqueda en páginas Web de contactos & $41.0 \%$ & $60.0 \%$ & $74.0 \%$ & $\chi^{2}=22.57 * * *$ \\
\hline Búsqueda de pareja sexual & $40.0 \%$ & $58.0 \%$ & $78.0 \%$ & $\chi^{2}=29.80 * * *$ \\
\hline $\begin{array}{l}\text { Insinuaciones sexuales con conocidos o } \\
\text { desconocidos }\end{array}$ & $55.0 \%$ & $77.0 \%$ & $85.0 \%$ & $\chi^{2}=24.11^{* * *}$ \\
\hline $\begin{array}{l}\text { Chatear reproduciendo fantasías sexuales } \\
\text { con otro usuario }\end{array}$ & $43.0 \%$ & $59.0 \%$ & $61.0 \%$ & $\chi^{2}=7.84^{*}$ \\
\hline Búsqueda de pareja romántica & $32.0 \%$ & $42.0 \%$ & $63.0 \%$ & $\chi^{2}=20.17 * * *$ \\
\hline $\begin{array}{l}\text { Contacto sexual por medio de Webcam } \\
\text { con otro usuario }\end{array}$ & $44.0 \%$ & $55.0 \%$ & $69.0 \%$ & $\chi^{2}=12.74 * *$ \\
\hline Respuesta a anuncios sexuales & $12.0 \%$ & $25.0 \%$ & $25.0 \%$ & $\chi^{2}=6.8^{*}$ \\
\hline Compra de material sexual online & $8 \%$ & $10 \%$ & $14 \%$ & $\chi^{2}=1.95$ \\
\hline Contacto con trabajadores/as sexuales & $17.0 \%$ & $19.0 \%$ & $12.0 \%$ & $\chi^{2}=1.93$ \\
\hline \multicolumn{5}{|l|}{ Motivaciones para la práctica del cibersexo } \\
\hline Distraerme & $56.0 \%$ & $46.0 \%$ & $68.0 \%$ & $\chi^{2}=9.88 * *$ \\
\hline $\begin{array}{l}\text { Encontrar material sexual con el que } \\
\text { masturbarme }\end{array}$ & $89.0 \%$ & $81.0 \%$ & $91.0 \%$ & $\chi^{2}=4.95$ \\
\hline $\begin{array}{l}\text { Disfrutar actividades sexuales que no } \\
\text { hago habitualmente }\end{array}$ & $50.0 \%$ & $44.0 \%$ & $35.0 \%$ & $\chi^{2}=4.65$ \\
\hline Aprender cosas sobre sexo & $49.0 \%$ & $45.0 \%$ & $46.0 \%$ & $\chi^{2}=0.34$ \\
\hline Relajarme del estrés de mis obligaciones & $62.0 \%$ & $58.0 \%$ & $58.0 \%$ & $\chi^{2}=0.44$ \\
\hline Animarme cuando estoy decaído & $32.0 \%$ & $24.0 \%$ & $32.0 \%$ & $\chi^{2}=2.05$ \\
\hline Conocer a otras personas & $20.0 \%$ & $24.0 \%$ & $46.0 \%$ & $\chi^{2}=18.66 * * *$ \\
\hline Intentar establecer relaciones & $13.0 \%$ & $13.0 \%$ & $31.0 \%$ & $\chi^{2}=14.03 * *$ \\
\hline
\end{tabular}

Nota: ${ }^{*} \mathrm{p}<0.05 ; * * \mathrm{p}<0.01 ; * * * \mathrm{p}<0.001$

\section{Actividad sexual online en función de la orientación sexual (mujeres)}

En mujeres, la orientación sexual sí influía sobre el porcentaje de participantes que en alguna ocasión habían utilizado Internet con fines sexuales ( $83 \%$ en heterosexuales frente al $94 \%$ en bisexuales y el $98 \%$ en homosexuales), y también sobre el tiempo invertido semanalmente en la ASO (siendo las bisexuales las que dedicaban una media semanal mayor -en torno a 1 hora y media frente a aproximadamente 1 hora en los otros casos-) (Tabla 2). En cuanto a su influencia sobre la prevalencia de distintas ASO, sólo 4 de las 12 exploradas mostraron prevalencias significativamente distintas en función de la orientación sexual. En este caso, el orden se invierte respecto a los resultados masculinos: excepto en el visionado de pornografía (donde la mayor prevalencia se daba entre mujeres homosexuales), en el resto de ASO las mujeres bisexuales y heterosexuales obtenían una prevalencia muy similar, y mayor que la de las mujeres homosexuales. Este es el caso por ejemplo de la búsqueda de información sexual (realizado por el 96\% de heterosexuales y el 95\% de bisexuales, frente al $77.6 \%$ de homosexuales; $p<$.001). En cuanto al uso de webcams con fin sexual, la prevalencia en bisexuales (41\%) superó sensiblemente la observada en heterosexuales (27\%) y homosexuales (10.2\%). En cuanto a motivación por consu- 
mir cibersexo, la orientación sexual influyó en 2 de ellas: el uso del cibersexo para relajarse del estrés ( $p=.010)$ y animarse cuando se está decaída ( $p=.027$ ). De nuevo, la prevalencia mayor se daba en mujeres bisexuales ( $51 \%$ y $19 \%)$.

Tabla 3. Comportamiento sexual online en función de la orientación sexual (mujeres)

\begin{tabular}{|c|c|c|c|c|}
\hline & $\begin{array}{l}\text { Heterosexual } \\
\text { es }\end{array}$ & $\begin{array}{c}\text { Bisexuale } \\
\mathrm{s}\end{array}$ & $\begin{array}{c}\text { Homosexua } \\
\text { les }\end{array}$ & $\begin{array}{l}\text { Estadístico } \\
\text { diferencial }\end{array}$ \\
\hline $\begin{array}{l}\text { ¿Alguna vez has utilizado Internet con } \\
\text { fines sexuales? }\end{array}$ & $83.0 \%$ & $94.0 \%$ & $98.0 \%$ & $\chi^{2}=10.99 * *$ \\
\hline $\begin{array}{l}\text { Tiempo medio dedicado a la ASO en } \\
\text { minutos }(D T)\end{array}$ & $\begin{array}{c}64.2 \\
(111.27)\end{array}$ & $\begin{array}{c}98.08 \\
(133.2)\end{array}$ & $54.7(58.6)$ & $f=3.24^{*}$ \\
\hline \multicolumn{5}{|l|}{ Tipos de actividad sexual online } \\
\hline $\begin{array}{l}\text { Visionado de imágenes o películas } \\
\text { pornográficas }\end{array}$ & $72.0 \%$ & $91.0 \%$ & $91.8 \%$ & $\chi^{2}=16.18 * * *$ \\
\hline $\begin{array}{l}\text { Búsqueda de información sobre } \\
\text { educación sexual }\end{array}$ & $96.0 \%$ & $95.0 \%$ & $77.6 \%$ & $\chi^{2}=17.26^{* * *}$ \\
\hline Lectura de textos eróticos & $69.0 \%$ & $80.0 \%$ & $63.3 \%$ & $\chi^{2}=5.50$ \\
\hline Búsqueda en páginas Web de contactos & $12.0 \%$ & $18.0 \%$ & $12.2 \%$ & $\chi^{2}=1.69$ \\
\hline Búsqueda de pareja sexual & $13.0 \%$ & $20.0 \%$ & $16.3 \%$ & $\chi^{2}=1.78$ \\
\hline $\begin{array}{l}\text { Insinuaciones sexuales con conocidos o } \\
\text { desconocidos }\end{array}$ & $59.0 \%$ & $57.0 \%$ & $34.7 \%$ & $\chi^{2}=8.67 *$ \\
\hline $\begin{array}{l}\text { Chatear reproduciendo fantasías } \\
\text { sexuales con otro usuario }\end{array}$ & $40.0 \%$ & $43.0 \%$ & $36.7 \%$ & $\chi^{2}=0.55$ \\
\hline Búsqueda de pareja romántica & $21.0 \%$ & $23.0 \%$ & $30.0 \%$ & $\chi^{2}=1.72$ \\
\hline $\begin{array}{l}\text { Contacto sexual por medio de Webcam } \\
\text { con otro usuario }\end{array}$ & $27.0 \%$ & $41.0 \%$ & $10.2 \%$ & $\chi^{2}=15.48^{* * *}$ \\
\hline Respuesta a anuncios sexuales & $2.0 \%$ & $8.0 \%$ & $4.1 \%$ & $\chi^{2}=3.99$ \\
\hline Compra de material sexual online & $11.0 \%$ & $7.0 \%$ & $8.2 \%$ & $\chi^{2}=1.02$ \\
\hline Contacto con trabajadores/as sexuales & $1.0 \%$ & $7.0 \%$ & $2.0 \%$ & $\chi^{2}=5.6$ \\
\hline \multicolumn{5}{|l|}{ Motivaciones para la práctica del cibersexo } \\
\hline Distraerme & $38.0 \%$ & $44.0 \%$ & $34.0 \%$ & $\chi^{2}=1.62$ \\
\hline $\begin{array}{l}\text { Encontrar material sexual con el que } \\
\text { masturbarme }\end{array}$ & $55.0 \%$ & $68.0 \%$ & $69.8 \%$ & $\chi^{2}=4.88$ \\
\hline $\begin{array}{l}\text { Disfrutar actividades sexuales que no } \\
\text { hago habitualmente }\end{array}$ & $29.0 \%$ & $27.0 \%$ & $18.9 \%$ & $\chi^{2}=1.91$ \\
\hline Aprender cosas sobre sexo & $56.0 \%$ & $46.0 \%$ & $43.4 \%$ & $\chi^{2}=2.96$ \\
\hline $\begin{array}{l}\text { Relajarme del estrés de mis } \\
\text { obligaciones }\end{array}$ & $30.0 \%$ & $51.0 \%$ & $43.4 \%$ & $\chi^{2}=9.25 * *$ \\
\hline Animarme cuando estoy decaído & $7.0 \%$ & $19.0 \%$ & $9.4 \%$ & $\chi^{2}=7.19^{*}$ \\
\hline Conocer a otras personas & $5.0 \%$ & $5.0 \%$ & $7.5 \%$ & $\chi^{2}=0.52$ \\
\hline Intentar establecer relaciones & $1.0 \%$ & $4.0 \%$ & $3.8 \%$ & $\chi^{2}=1.92$ \\
\hline
\end{tabular}

Nota: ${ }^{*} \mathrm{p}<0.05 ;{ }^{* *} \mathrm{p}<0.01 ; * * * \mathrm{p}<0.001$

\section{DISCUSIÓN Y CONCLUSIONES}

El objetivo de esta investigación era analizar si el consumo de cibersexo (más en concreto, su frecuencia, el tipo de ASO realizadas y los motivos que explican su consumo) diferían en función de la orientación sexual. A tal fin, 553 participantes, clasificados de acuerdo con su sexo y orientación sexual, completaron una encuesta sobre comportamiento sexual online. En hombres, la principal conclusión es que la orientación sexual no influiría sobre la frecuencia de consumo de cibersexo (es decir, el tiempo semanal dedicado a la ASO), pero sí sobre la forma 
(tipo de ASO realizadas) y los motivos que justificarían su consumo (aunque de forma mucho más limitada). En cuanto al tipo de ASO realizadas, los hombres homosexuales (seguidos muy de cerca por los bisexuales) muestran mayor prevalencia de prácticamente todas las ASO. Esto sugeriría que los hombres con una orientación sexual alternativa a la heterosexual encontrarían en Internet el recurso que les permite expresarse sexualmente, sobre todo en lo que se refiere a conocer a otras personas; así, las mayores diferencias las encontramos precisamente en aquellas ASO que implicarían la interacción con otros usuarios, tanto en lo que se refiere a las que facilitarían la satisfacción sexual inmediata online (plasmado en el porcentaje de los que usan chats o webcams sexuales) como aquellas que facilitarían el establecer relaciones románticas o sexuales offline. Estas cifras superan ampliamente las obtenidas en la población general (Ballester-Arnal et al., 2020), pero quedan lejos de los porcentajes obtenidos entre otros estudios con población HSH en EEUU (Rosser et al., 2009). Como se planteaba en la introducción, sería plausible que el entorno cultural justifique estas diferencias: mientras que en EEUU, la actitud hacia la homosexualidad todavía sustenta un cierto rechazo, España se sitúa en cabeza en cuanto a aceptación de la homosexualidad (Petrou \& Lemke, 2018). Desde esta perspectiva, el mayor rechazo social podría actuar incrementando el consumo de cibersexo como medio seguro de satisfacción sexual (hipótesis del «oasis erótico» [Ross et al., 2000]), mientras que en contextos más abiertos, no sería tan necesario recurrir a la esfera digital. El hecho de que, en nuestro estudio, las motivaciones para consumir cibersexo apenas difirieran en función de la orientación sexual apoyaría esta hipótesis: así, los motivos que conducen al consumo de cibersexo en heterosexuales, bisexuales y homosexuales son prácticamente iguales. La única excepción se aprecia en cuanto al consumo de cibersexo como forma de entablar relaciones, lo que sugeriría más bien que el cibersexo es una actividad preferente para ligar más por su comodidad (facilita encontrar a gente que comparte una determinada preferencia sexual) que por cualquier otro motivo (Grov et al., 2008).

En mujeres, las conclusiones cambian notablemente. En este caso, el consumo más activo (p.e., en términos de dedicación temporal) se daría entre las bisexuales, mientras que las homosexuales muestran una ASO mucho más moderada (incluso que las heterosexuales). Asimismo, el impacto de la orientación sexual sobre la realización de distintas ASO sería muy modesto, y en los casos en los que sí influye, nuevamente las bisexuales son las que mostrarían un consumo más activo (por ejemplo, en cuanto al uso de la webcam con fines sexuales). Estos resultados sugerirían que, en mujeres, el cibersexo podría dibujarse como forma de satisfacer los deseos sexuales no cubiertos de las bisexuales (presumiblemente, fantasías sexuales correspondientes al sexo contrario de la persona con las que mantengan relaciones), pero sólo podríamos plantearlo como conjetura (la naturaleza de este estudio no nos permite plantear una explicación más contrastada). En cuanto a las motivaciones, la orientación sexual de las mujeres sólo influyó en las dos relacionadas con la regulación emocional: así, las bisexuales lo utilizaban más para relajarse del estrés o bien cuando se encontraban decaídas. La presencia de estas dos motivaciones normalmente correlacionan con un uso problemático del cibersexo (Lew-Starowicz et al., 2019) de modo que en futuros estudios, convendría analizar si el riesgo de consumo adictivo es mayor entre mujeres bisexuales. Asimismo, las notables diferencias en el consumo de cibersexo en mujeres homosexuales (sobre todo en comparación con los hombres homosexuales) justificaría la realización de estudios que permitan acercarse a la construcción de su identidad online (prioritariamente, a través de metodología cualitativa).

\section{REFERENCIAS BIBLIOGRÁFICAS}

Ballester-Arnal, R., Castro-Calvo, J., García-Barba, M., Ruiz-Palomino, E., \& Gil-Llario, M. D. (2020). Online Sexual Activities (OSAs) across the lifespan: a large-scale cross-sectional study [manuscript submitted for publication]. Departamento de Psicologiía Básica, Clínica y Psicobiología, Universitat Jaume I.

Castro-Calvo, J., Giménez-García, C., Gil-Llario, M. D., \& Ballester-Arnal, R. (2018). Motives to engage in Online Sexual Activities and their links to an excessive and problematic use: a Systematic Review. Current Addiction Reports, 5(4), 491-510. https://doi.org/https://doi.org/10.1007/s40429-018-0230-y

Cooper, A., \& Griffin-Shelley, E. (2002). The internet:The next sexual revolution. In A. Cooper (Ed.), Sex \& the internet: A guidebook for clinicians (pp. 1-15). Brunner-Routledge. 
Daneback, K., Sevcikova, A., Mänsson, S.-A., \& Ross, M. W. (2013). Outcomes of using the internet for sexual purposes: fulfilment of sexual desires. Sexual Health, 10(1), 26-31. https://doi.org/10.1071/SH11023

Grov, C., Bamonte, A., Fuentes, A., Parsons, J. T., Bimbi, D. S., \& Morgenstern, J. (2008). Exploring the internet's role in sexual compulsivity and out of control sexual thoughts/behaviour: A qualitative study of gay and bisexual men in New York City. Culture, Health and Sexuality, 10(2), 107-125. https://doi.org/10.1080/13691050701564678

Lew-Starowicz, M., Lewczuk, K., Nowakowska, I., Kraus, S., \& Gola, M. (2019). Compulsive Sexual Behavior and Dysregulation of Emotion. Sexual Medicine Reviews, 1-15. https://doi.org/10.1016/j.sxmr.2019.10.003

Petrou, P., \& Lemke, R. (2018). Victimisation and life satisfaction of gay and bisexual individuals in 44 European countries: the moderating role of country-level and person-level attitudes towards homosexuality. Culture, Health and Sexuality, 20(6), 640-657. https://doi.org/10.1080/13691058.2017.1368710

Ross, M. W., Tikkanen, R., \& Mansson, S. (2000). Differences between Internet samples and conventional samples of men who have sex with men: implications for research and HIV interventions. Social Science \& Medicine, 51, 749-758.

Rosser, B. R., Miner, M. H., Bockting, W. O., Ross, M. W., Konstan, J., Gurak, L., Stanton, J., Edwards, W., Jacoby, S., Carballo-Diéguez, A., Mazin, R., \& Coleman, E. (2009). HIV risk and the Internet: Results of the men's internet sex (MINTS) study. AIDS and Behavior, 13(4), 746-756. https://doi.org/10.1007/s10461-008-93998

Seal, D. W., Benotsch, E. G., Green, M., Snipes, D. J., Bull, S. S., Cejka, A., Lance, S. P., \& Nettles, C. D. (2015). The Use of Internet Chat Rooms to Meet Sexual Partners: A Comparison of Non-Heterosexually Identified Men with Heterosexually Identified Men and Women. International Journal of Sexual Health, 27(1), 1-15. https://doi.org/10.1080/19317611.2014.918921

Shaughnessy, K., Byers, E. S., \& Walsh, L. (2011). Online Sexual Activity Experience of Heterosexual Students: Gender Similarities and Differences. Archives of Sexual Behavior, 40(2), 419-427.

Wéry, A., \& Billieux, J. (2017). Problematic cybersex: Conceptualization, assessment, and treatment. Addictive Behaviors, 64, 238-246. https://doi.org/10.1016/j.addbeh.2015.11.007 
